\title{
Fractal Generation From the "Luque Method" for Simplification of Logic Functions
}

By David Luque Sacaluga

A new way to simplify of logic functions.

This project presents a new method for the simplification of logic functions or truth tables. An example of the application of this method is shown and compared to the Karnaugh method.

In addition, and as a consequence of this new method, a fractal is generated that can show a truth table for infinite variables.

\section{Description of Method}

Based on the cyclical character of Gray code, I take a circumference and divide it in $2^{\mathrm{n}}$ equal parts, $n$ being the number of variables.

I assign an a, b, c, d... etc., variable to each axis or axes that divide the circumference in 2, 4, 8, 16... etc., equal parts, as can be seen on Figures 1, 2, and 3.

Assigning a binary code to the obtained sectors so that they are ordered according to Gray code, I get the representation on Figure 2.

This is the way to represent a Truth Table applying the Luque Method.

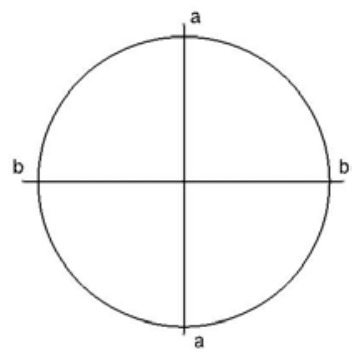

Figure 1: Circumference divided into 4 sectors when $\mathrm{n}=2$. Axes $\mathrm{a}$ and $\mathrm{b}$.

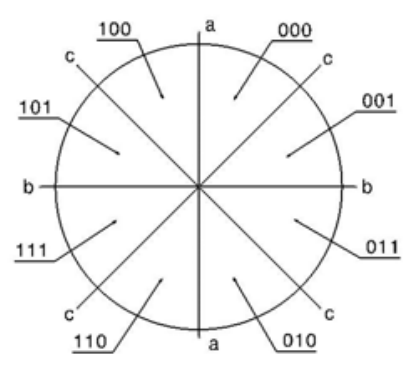
Figure 2: Circumference divided into 8 sectors when $n=3$. Axes $\mathrm{a}, \mathrm{b}$, and c. Representation according to C. Gray.

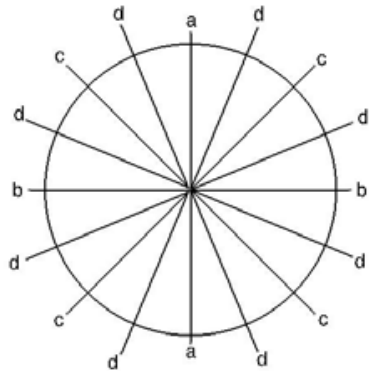

Figure 3: Circumference divided into 16 sectors when $\mathrm{n}=4$. Axes $\mathrm{a}, \mathrm{b}, \mathrm{c}$ and $\mathrm{d}$

I separate each of the variables and get Figures 4, 5, 6 and 7 where the representation of the 4 variable has been used as an example. 
The a-variable divides the circumference into two sectors, one for $\mathrm{a}=0$ and another one for $\mathrm{a}=1$ (Figure 4 ). The red shadowed-section contains the values of $\mathrm{a}=1$ and the nonshadowed-section those of $a=0$.

The $b$-variable also divides the circumference into two sectors, one for $b=1$ and another one for $b=0$, (Figure 5). Together with the a-variable, it divides the circumference into four equal sectors. The blue shadowed-section contains the values of $b=1$ and the nonshadowed-section those of $b=0$.

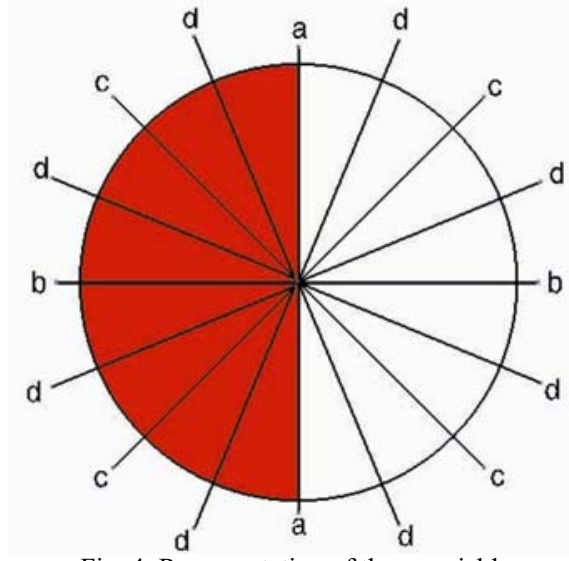

Fig. 4. Representation of the a-variable.

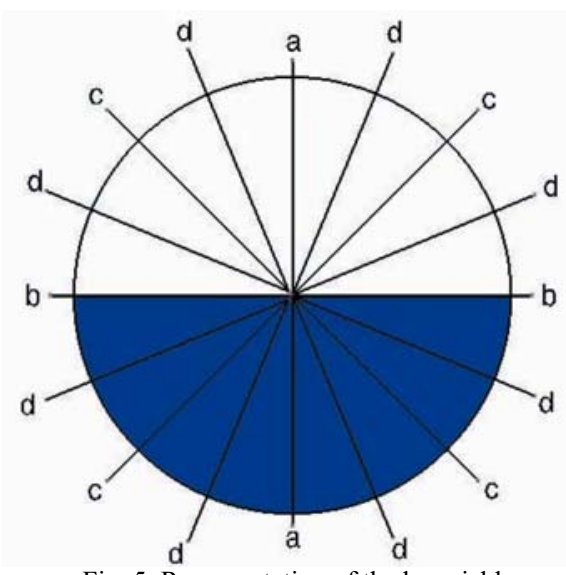

Fig. 5. Representation of the b-variable.

The c-variable divides the circumference into four sectors, two for $\mathrm{c}=1$ and two for $\mathrm{c}=0$ (Figure 6). Together with the a- and b-variables it divides the circumference into 8 sectors. The colour shadowed-sections contain the values of $\mathrm{c}=1$ whereas the other nonshadowed-section give the values of $\mathrm{c}=0$.

The d-variable divides the circumference in eight sectors, 4 for $d=1$ and 4 for $d=0$ (Figure 7). Together with the a-, b-, and c-variables it divides the circumference into 16 sectors. The green shadowed-sections contain the values of $d=1$ and the non-shadowedsections those of $d=0$.

Fig. 6. Representation of the c-variable.

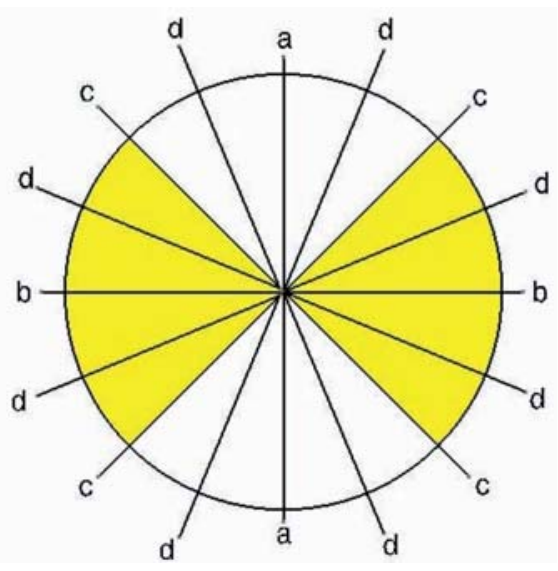

Fig. 7. Representation of the d-variable.

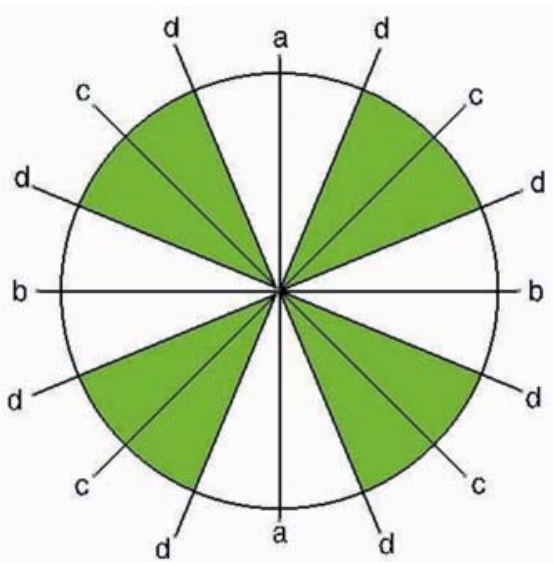


Table 1: Truth table with three variables.

\begin{tabular}{|c|c|c|c|c|}
\hline $\mathrm{a}$ & $\mathrm{b}$ & $\mathrm{c}$ & $\mathrm{F}$ & $\begin{array}{l}\text { For more variables I observed that the process would be the } \\
\text { same, knowing that the sectors into which the circumference }\end{array}$ \\
\hline 0 & 0 & 0 & 1 & \\
\hline 0 & 0 & 1 & 0 & Beginning with the Truth Table, I will mark the sectors \\
\hline 0 & 1 & 0 & 1 & containing 1 and will leave those containing 0 blank. \\
\hline 0 & 1 & 1 & 1 & Then I look for the symmetries between the axes of the \\
\hline 1 & 0 & 0 & 0 & sectors whose value is 1 . \\
\hline 1 & 0 & 1 & 0 & I start looking at the symmetries with regard to the last \\
\hline 1 & 1 & 0 & 0 & variable, that is to say, the most repetitive, leaving for the \\
\hline 1 & 1 & 1 & 1 & $\begin{array}{l}\text { end the a- and b-variables (vertical and horizontal axes } \\
\text { respectively). }\end{array}$ \\
\hline
\end{tabular}

With the exception of the symmetries in the a- and b-axes, the other symmetries will only be validated if they exist in the same quadrant.

When the symmetry exists, the symmetrical or variable axis disappears from the term of the equation, in this way simplifying the logic function.

\section{Sample Application}

Given Table 1, I will get the simplified function applying Karnaugh and Luque.

\begin{tabular}{|c|c|c|c|c|}
\hline$c^{a b}$ & 00 & 01 & 11 & 10 \\
\hline 0 & 1 & 1 & 0 & 0 \\
\hline 1 & 0 & 1 & 1 & 0 \\
\hline
\end{tabular}

Table 2: Karnaugh Table out of Table 1. 
The process followed by Karnaugh to obtain the simplified function is shown on Table 2 . The function represented by the Truth Table is shown in the [1]-equation.

$$
F=\bar{a}\langle\bar{c}+b<c
$$

The circle obtained applying Luque Method is shown on Figure 8.

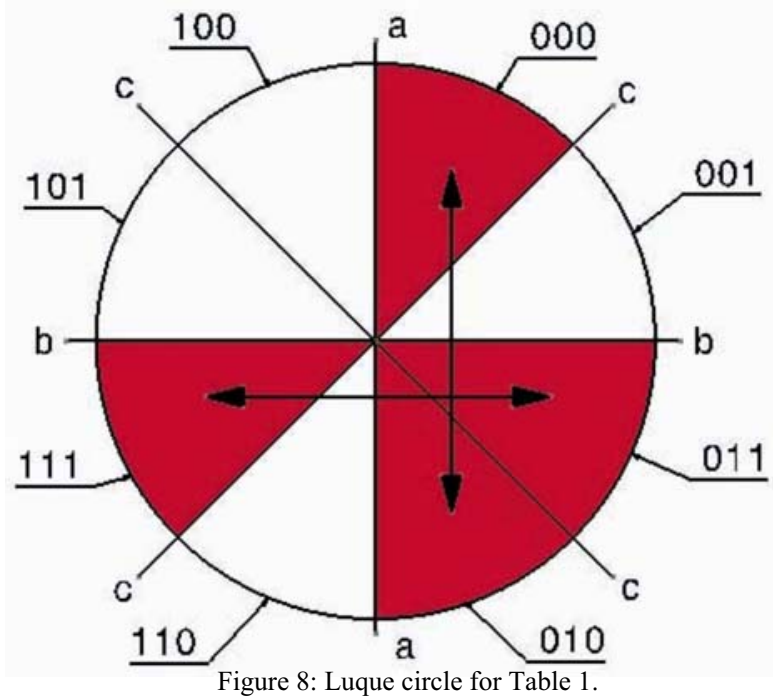

I observe symmetry with regard to the b-axis, terms 000 and 010 ; then this variable disappears and the first term of the function will be: $\bar{a}<\bar{c}$

The second term is symmetrical with regard to the a-axis, 111 and 011 ; then I will eliminate the a-variable, so I will have: $b\langle c$

$$
F=\bar{a}<\bar{c}+b<c
$$

The [2]-equation is the one obtained applying the Luque Method, which coincides with the [1]-equation obtained with the Karnaugh Method.

\section{Fractal Generation}

I have shown how the variables can be presented as a-variable (Fig. 4), b-variable (Fig. 5), c-variable (Fig. 6), and so on.

If multiple variables are assumed, each one would have a representation in the Luque Circle, and in addition the following conditions would be fulfilled: 


$$
\begin{aligned}
& \bigcup_{1}^{\times} a_{i}=\bigcup \\
& \bigcap_{1}^{\times} a_{i}=\Phi
\end{aligned}
$$

Being $a_{i}$ each one of the variables.

The collection of all variables is equal to the total set and the intersection of all variables is equal to null set.

To explain this better one can rely on Venn Diagrams.

a $\mathrm{U} b$ would be in $3 / 4$ parts of the circumference.

a U b U c would be in $7 / 8$ parts of the circumference.

a U b U c U d would be in 15/16 parts of the circumference.

Calculating we would have the limit of the expression [5]:

$$
\lim _{x \rightarrow x} \frac{2^{x}-1}{2^{x}}=1
$$

Now, let's see what happen with the intersection.

$a_{-}$B would be in $1 / 4$ parts of the circumference.

$\mathrm{A}_{-} \mathrm{B} \_\mathrm{C}$ would be in $1 / 8$ parts of the circumference.

A_B_C_ D would be in $1 / 16$ parts of the circumference.

Calculating we would have the limit of the expression [6]:

$$
\lim _{x \rightarrow x} \frac{1}{2^{x}}=0
$$

Figure 9 shows a new representation for only one variable, in this case "a", where one can see the areas where $\mathrm{a}=1$ and $\mathrm{a}=0$.

Figure 10 shows the representation for two variables ("a" and "b") where one can see the areas where $\mathrm{a}=1, \mathrm{a}=0, \mathrm{~b}=1, \mathrm{~b}=0$. 


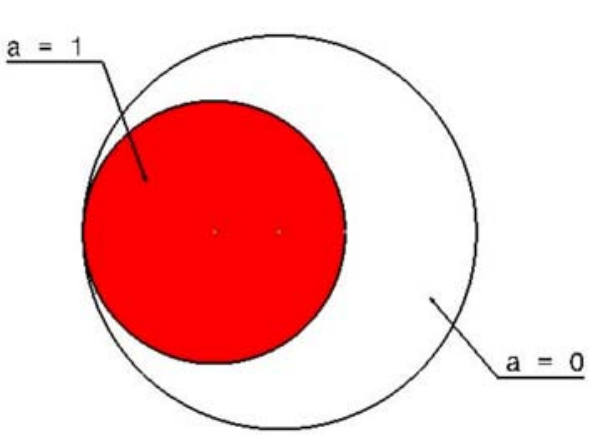

Figure 9: Representation of one variable.

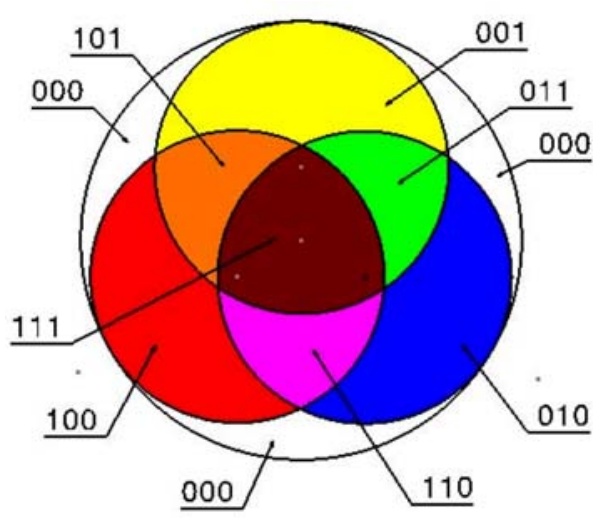

Figure 11: Representation of three variables.

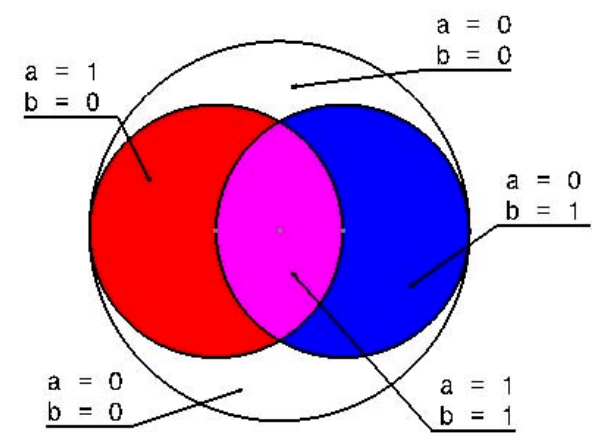

Figure 10: Representation of two variables.

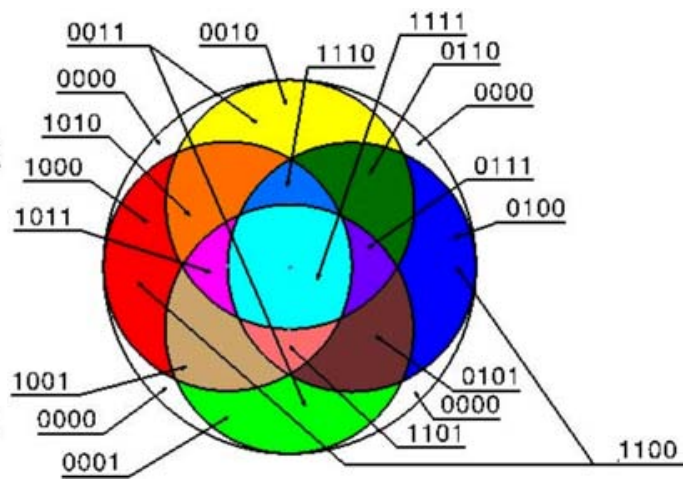

Figure 12: Representation of four variables.

Figure 11 shows the representation for three variables $\mathrm{a}, \mathrm{b}$ and $\mathrm{c}$.

Figure 12 shows the representation for four variables a, b, c and d.

What happen when we reach infinite?

If we reach infinite, the conditions [3] and [4] will be fulfilled.

In Figure 13 we can see the representation for the condition [3], where only four variables are represented. When there are infinite variables the circumference should be completely shadowed and would fulfil condition [3].

In Figure 14 we can see the representation for condition [4], where only four variables are represented. When there are infinite variables we would have a shadowed circumference 
in the centre which would be the intersection of all variables. Then, the condition 4 would be not fulfilled.

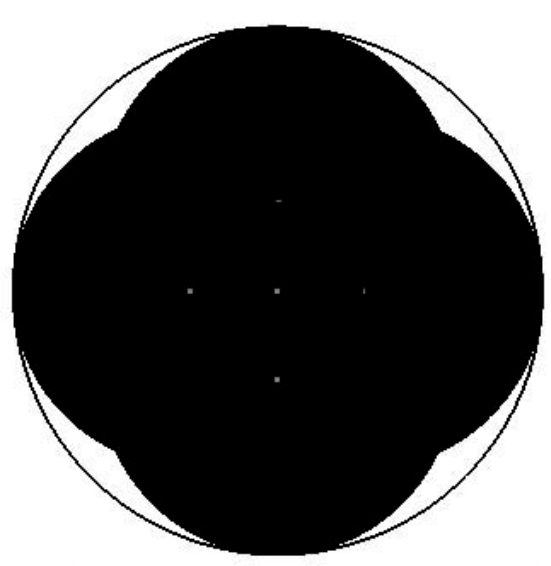

Figure 13: Representation for the condition [3] for four variables.

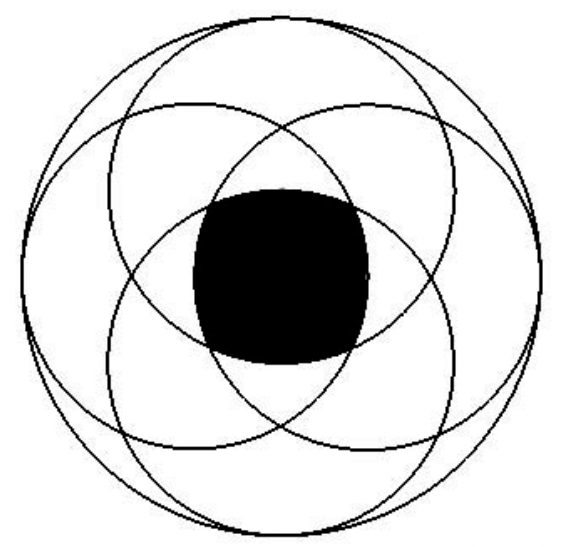

Figure 14: Representation for the condition [4] for four variables

To fulfil the condition [4], in the circumference generated due to intersection, I introduce the same system, as can be appreciated on Figures 15, 16 and 17.

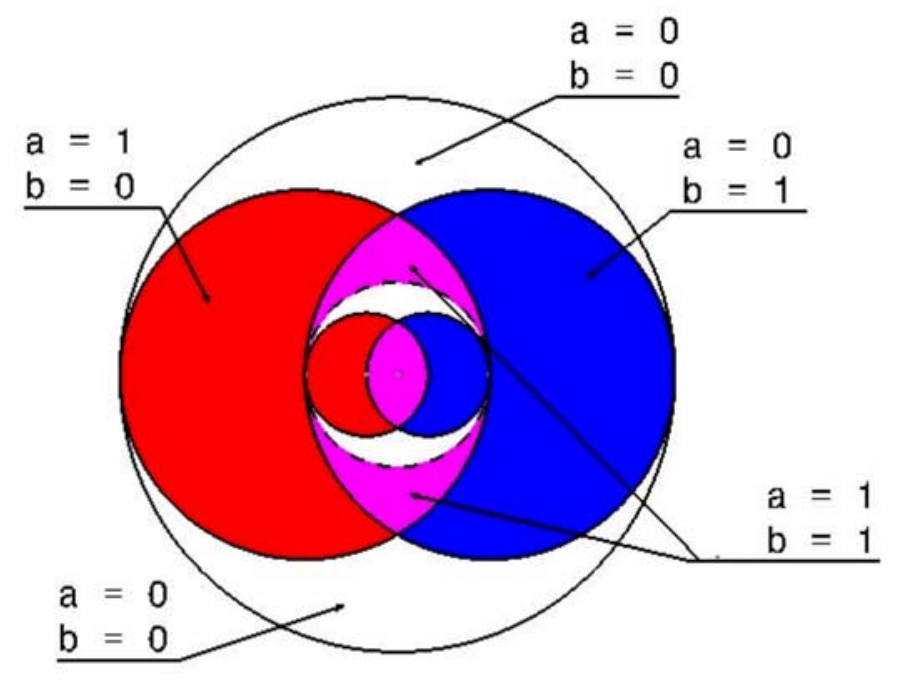

Figure 15: Representation for two variables 

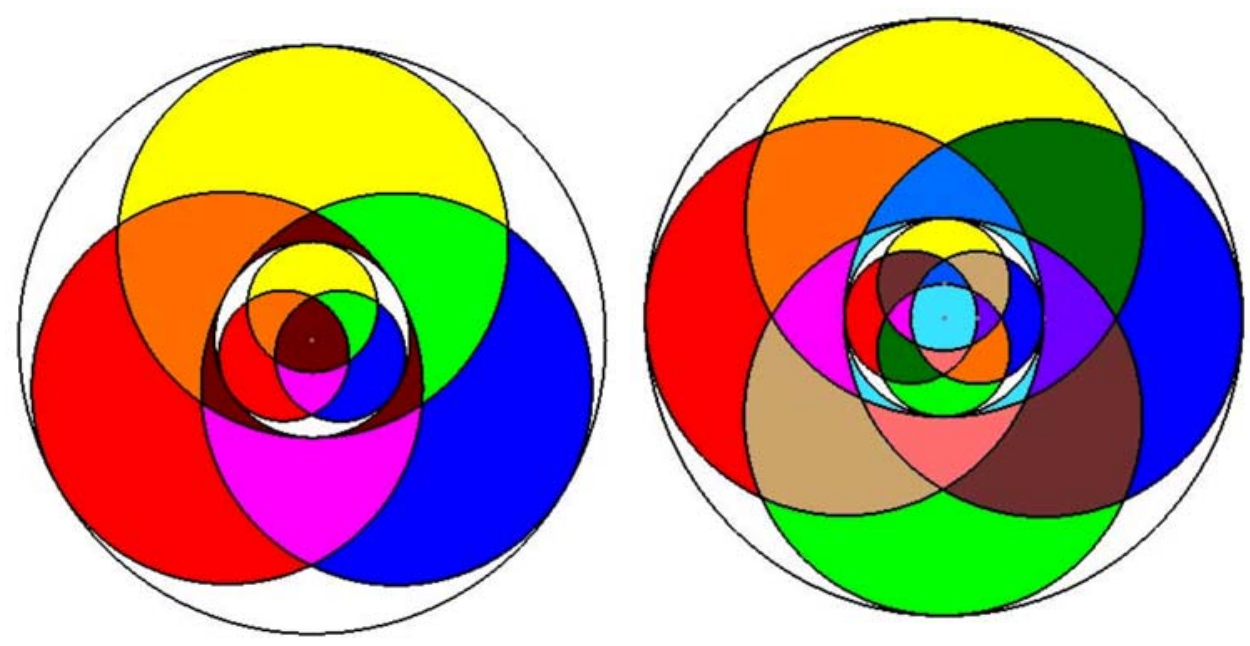

For infinite variables the condition [3] is fulfilled, however condition [4] is not satisfied, as we would have a circumference in the center again. To fulfil the condition [3] the same process would be repeated untill infinite. Conditions [3] and [4] are satisfied by this way.

A fractal is generated as shown on Figure 18 which is repeated to infinite itself.

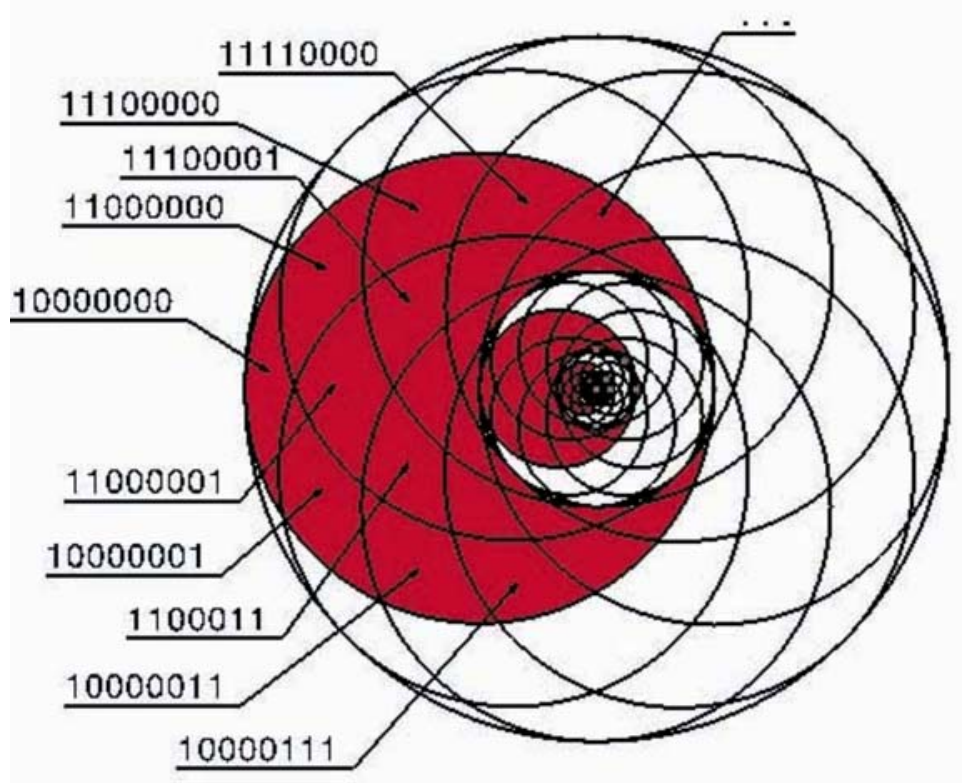

Figure 18: Fractal representation of the a-variable for a system of eight variables. 


\section{Conclusion}

The Luque Method presented in this project can substitute for the Karnaugh Method as a good alternative for electronics students. It is also interesting to see the disposition of the Bistables using this method as it is easier to memorise.

A representation of a truth table for infinite variables can be obtained due to the new disposition through fractals.

The information presented above has potential for application in the electronics and automation fields. Further study is necessary.

\section{References}

[1] Gonzalez R, Moreno F, Romero E. (1990). Sistemas Electrónicos Digitales, Universidad de Cádiz, Cádiz.

[2] Mandado E. (1998). Sistemas Electrónicos Digitales Tomo I, Marcombo, Barcelona.

[3] Mandelbrot B. (1997). La geometría fractal de la naturaleza, Tusquets Editores, Barcelona.

David Luque Sacaluga is a program designer for Airbus S.L. He studied electronic engineering at the University of Cadiz, Spain, and has previously published papers on fractal generation and chaos. 